Health \& Medicine | Alexander Lorenz

\section{Candida auris}

The path of yeast resistance

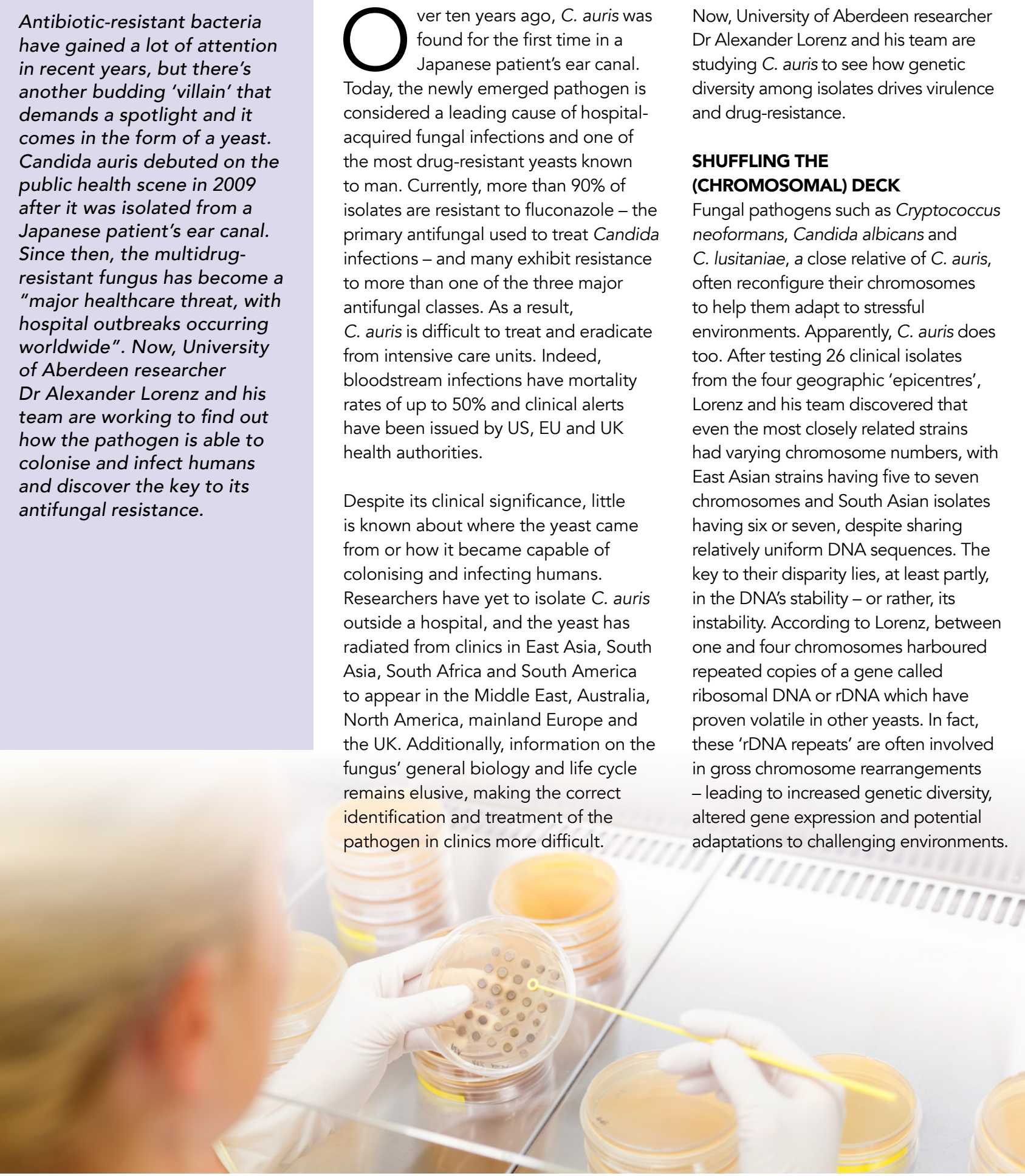
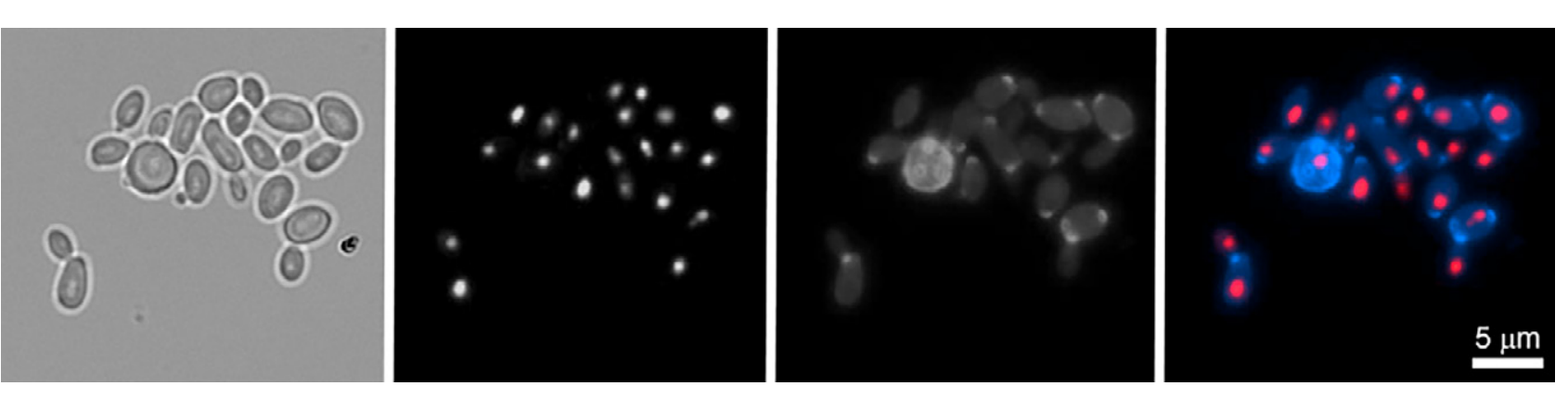

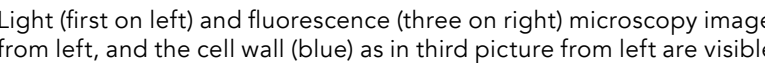

To see just how helpful these

chromosome rearrangements can be,

Lorenz and his team subjected four

clinical strains (each from a different

geographical hotspot) to heat, osmotic

and genotoxic stress. To induce osmotic

stress, the researchers exposed $C$. auris to asmotic stress is the sudd sugar sorbose. Wuter merss is the suddon change in (cter movent across he cell wall (or to high concentrations of sugars or salts. Cells can adapt to this via stress response mechanisms. This process mimics the consequences of treating yeast with an echinocandin-type antifungal, which prevents cell wall synthesis. Meanwhile, genotoxic stress was achieved using a chemical that reduces the number of nucleotides available for DNA replication. Finally, heat stress was produced by growing the fungus at a sweltering $42^{\circ} \mathrm{C}$.

In all cases, chromosomes appeared, disappeared or changed sizes, with equency and type of alteration differing deped Howeververy few modifiction used. Howeververy few modifications American strains - actually resulted in

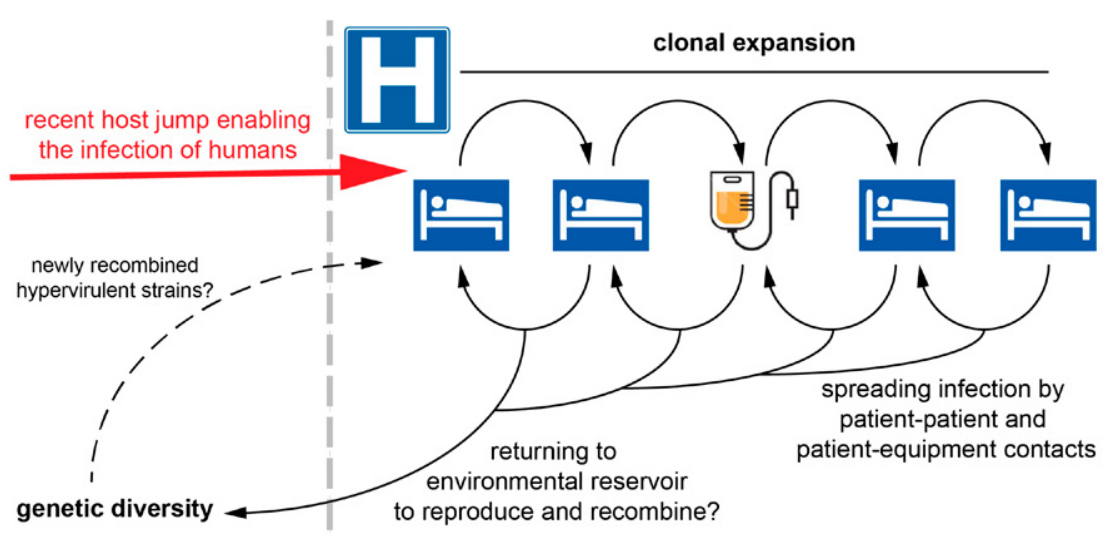

Environment Hospital improved growth. It's worth noting though, that they did not appear

This is especially striking in the South African strain, which continued to grow after consolidating its genome from seven to three chromosomes when chromosomes from end-to-end or breaking its chromosomes apart and fusing the pieces to other chromosomes, according to the researchers. Such drastic changes could provide opportunities for

\section{A SELECTION OF TRICKS}

Chromosome shuffling isn't the only adaptive trick up $C$. auris'sleeve. The pathogenic yeast is studded with likely achieved this, either by tethering

Since [2009], the multidrug-resistant fungus has become a 'major healthcare threat, with hospital outbreaks occurring worldwide'.

transporter proteins, capable of pumping antifungal drugs out of the cell. These in the presence of antifungals such as azoles and polyenes, bolstering the yeast's defences against the distressing compounds.

Additionally, under certain genotoxic conditions, many C. auris strains are capable of forming filaments called pseudohyphae. While there is insufficient data to confirm the role of pseudohypha in the virulence of $C$. auris, filamentous growth has been shown to play an important part in host-pathogen attachment and disease developmen in other fungi. Combined with the malleable and adaptable genome of colates to ham fild allow clinical during pathogenesis.

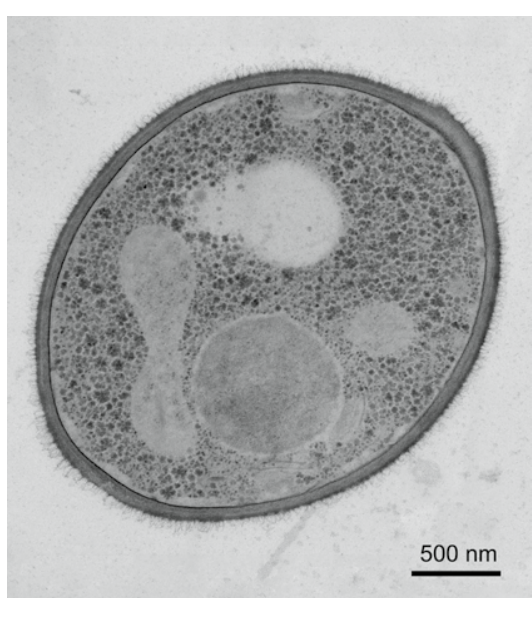

Electron microscopy image of $C$. auris 


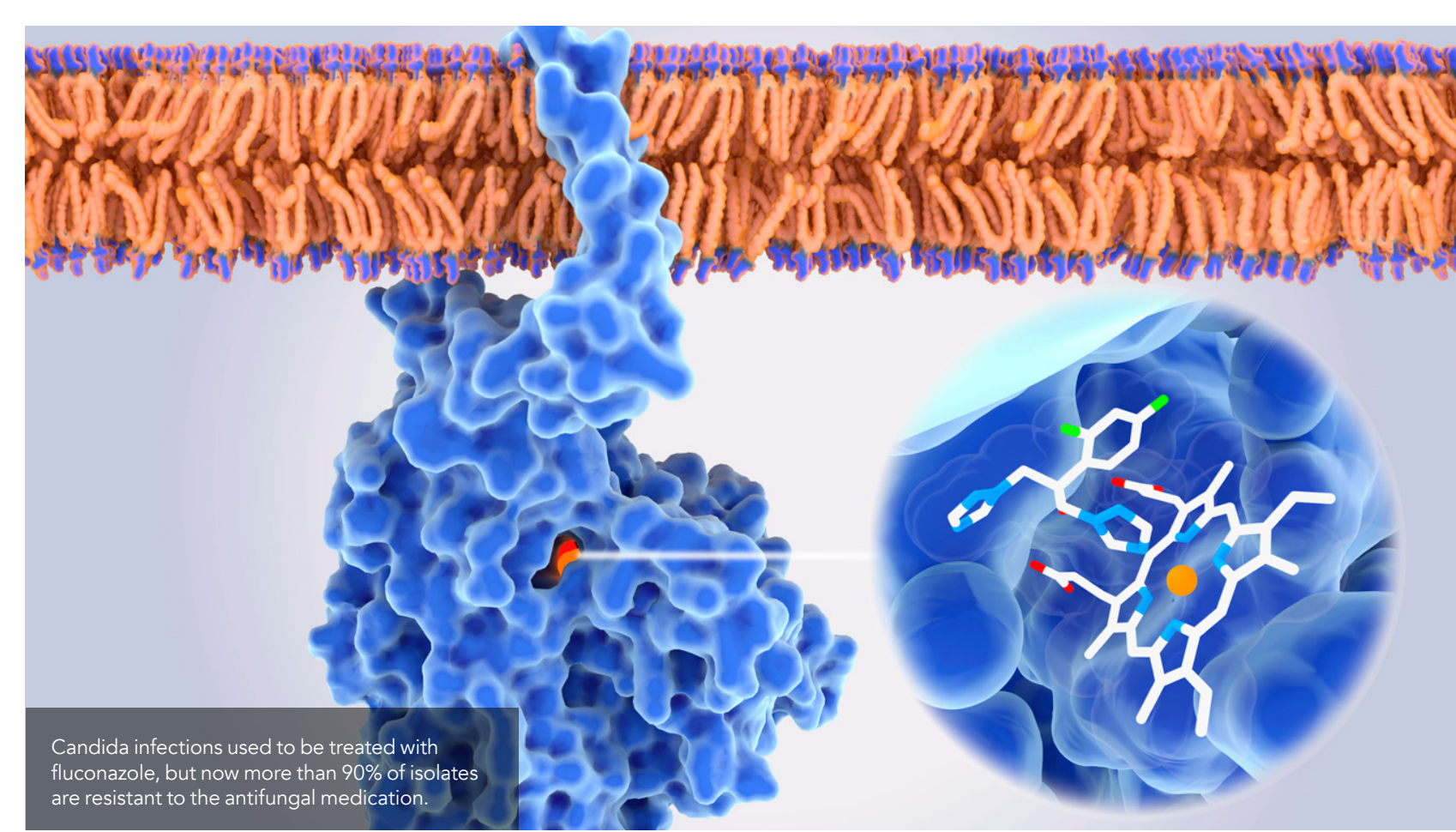

... even the most closely related strains had varying chromosome numbers .. despite sharing relatively uniform DNA sequences.

\section{RESEARCHERS SHOW THEIR HAND} While $C$. auris has shown itself to be a

Lorenz and his team have found potential way to fight back - at least formidable and 'mysterious' organism in the clinic. Instead of an antifunga

Microevolution Experiment

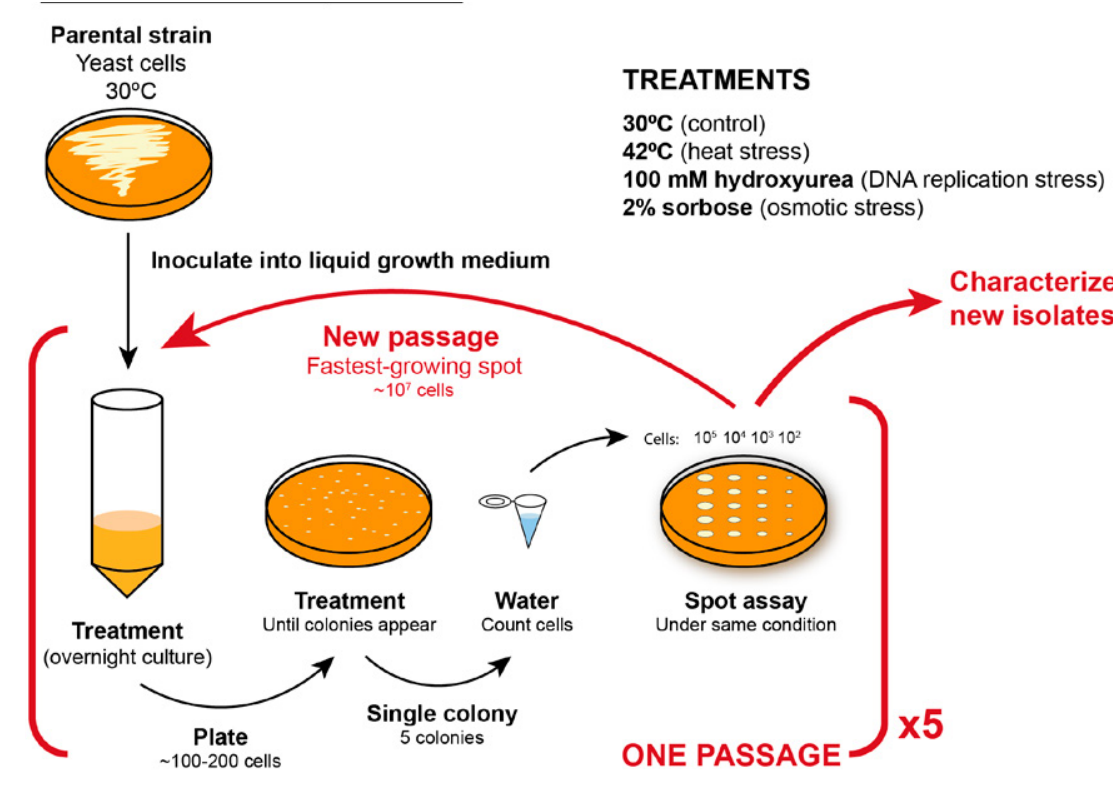

drug, however, the researchers used another fungus. When cultured together with $C$. auris, a predatory yeast, Saccharomycopsis schoenii, attacked S. schoenii had eliminated $34 \%$ of $C$. auris, while almost no $C$. auris cells died drug-resistant forms of $C$. auris were still susceptible to the predator. The results

\section{NEXT STEPS}

In the 11 years since its discovery, co the bignest causes of hospitatacquired fungal infections and one of the most drug-resistant yeasts known to science. Despite its global prevalence and dangerous nature, little is known about where the fungus came from, how it came to infect humans or how it help fill these knowledge gaps, Lorenz and his team have been studying C. auris - exploring its adaptability genome structure, virulence factors and even ways to combat the fungus in clinics. Already, they've made considerable progress, but there's still a lot left to learn its gent this mysterious organism, including its general biology, life cycle, addition and how to defeat the yeast in patients. ance-like structures. Within six hours, when cultured on its own. Importantly, make S. schoenii a promising, if novel, developed antifungal resistance. To

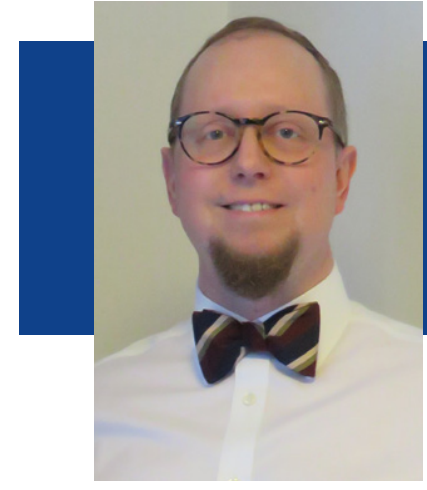

\section{Behind the Research}

\section{Dr Alexander Lorenz}

: a.lorenz@abdn.ac.uk T: $+441224437323 \quad$ W: https://www.abdn.ac.uk/ims/profiles/a.lorenz W: https://homepages.abdn.ac.uk/a.lorenz/pages/index.html W: $\mathbf{h t t p s : / / t w i t t e r . c o m / L o r e n z L a b ~}$ W: https://www.researchgate.net/profile/Alexander_Lorenz2

\section{Research Objectives}

Dr Lorenz's work investigates how genetic diversity is Denerated in Candida auris, and how this drives the evolution of antifungal resistance.

\section{References}

- Cullen, P. and Sprague Jr, G. (2012). The Regulation of Filamentous Growth in Yeast. Genetics, [online] 190(1), 23-49. Available at: https://www.ncbi.nlm.nih.gov/pmc/

Gubbins, P. and Anaissie, E. (2009). Antifungal therapy. Clinical Mycology (Second Edition), [online] 161-195. Available at. Atpss/165w.sciencedirect.com/science/

- Junker, K., Bravo Ruiz, G., Lorenz, A., Walker, L., Gow, Saccharomycopsis schoenij predates and kills multi-drug resistant Candida auris. Scientific Reports, [onlinel 8 . Available at: https://www.nature.com/articles/s41598018-33199-z

- Bravo Ruiz, G., Ross, Z., Gow, N. and Lorenz, A. (2020). Pseudohyphal growth of the emerging pathogen Candida auris is triggered by genotoxic stress throug the S phase checkpoint. mSphere, [online] 5(2), e00151

- Bravo Ruiz, G., Ross, Z., Holmes, E., Schelenz, S., Gow, $N$. and Lorenz, A. (201). Rapid and extensive karyotype Current Genetics, [onlinel 65(5), 1217-1228. Availables. https://link.springer.com/article/10.1007\%2Fs00294019-00976-w

- Wasi, M. Khandelwal N., Moorhouse, A., Nair, R. Vishwakarma, P., Bravo Ruiz, G., et al. (2019). ABC Transporter Genes Show Upregulated Expression in Drug-Resistant Clinical lsolates of Candida auris A Genome-Wide Characterization of ATP-Binding Microbiology, [online] 10. Available at: https://www. frontiersin.org/articles/10.3389/fmich. 2019.01445/full - Yang, F., Kravets, A., Bethlendy, G., Welle, S. and
Rustchenko, E. (2013). Chromosome 5 Monosomy of Candida albicans Controls Susceptibility to Various Toxic Agents, Including Major Antifungals. Antimicrobia Agents and Chemotherapy, lonline] 57(10), 5026 36. Available at: https://www.ncbi.nlm.nih.go

\section{Detail}

Alexander Lorenz
Institute of Medical Sciences (IMS)

University of Aberdeen

Aberdeen AB25 2ZD

Aberdeen AB25
United Kingdom

Bio

Alexander Lorenz joined the University of Aberdeen, UK as a lecturer in 2013. He graduated (2000) and earned a years before moving to the University of Oxford, UK as a research fellow.

Funding

(212524/Z/18/Z) (MR/P501955/1)

Collaborators
Gustavo BRAVO RUIZ

\section{Personal Response}

What prompted you to study C. auris?

II My main reason was being curious about the weird to Aberdeen in 2013 with a view to collaborate with researchers at the Aberdeen Fungal Group on a project with a pathogenic fungus. Professor Neil Gow then suggested that studying the life cycle of $\mathrm{C}$. auris would relevant, and we know so little about it.

Do you plan to continue studying the yeast, and if so, have you got any ideas for your next project?

My lab is working hard to develop molecular cell biology tools and genetic manipulation techniques for $C$. auris to do more in-depth experiments. Using genomics, we continue to study the strain with only its chromosomes during stress response. 